Kansas State University Libraries

New Prairie Press

\title{
STRATEGIES FOR REDUCING CONTROL GROUP SIZE IN EXPERIMENTS USING LIVE ANIMALS
}

\author{
Matthew Kramer \\ USDA, Agricultural Research Service, matt.kramer@ars.usda.gov \\ Enrique Font \\ Instituto Cavanilles de Biodiversidad y Biología Evolutiva, Universidad de Valencia, enrique.font@uv.es
}

Follow this and additional works at: https://newprairiepress.org/agstatconference

Part of the Agriculture Commons, Applied Statistics Commons, Laboratory and Basic Science Research Commons, and the Pharmacology, Toxicology and Environmental Health Commons

\section{(c) (1) $\Theta($}

This work is licensed under a Creative Commons Attribution-Noncommercial-No Derivative Works 4.0 License.

\section{Recommended Citation}

Kramer, Matthew and Font, Enrique (2016). "STRATEGIES FOR REDUCING CONTROL GROUP SIZE IN EXPERIMENTS USING LIVE ANIMALS," Conference on Applied Statistics in Agriculture. https://doi.org/ $10.4148 / 2475-7772.1479$

This Event is brought to you for free and open access by the Conferences at New Prairie Press. It has been accepted for inclusion in Conference on Applied Statistics in Agriculture by an authorized administrator of New Prairie Press. For more information, please contact cads@k-state.edu. 


\title{
STRATEGIES FOR REDUCING CONTROL GROUP SIZE IN EXPERIMENTS USING LIVE ANIMALS
}

\author{
Matthew Kramer ${ }^{1}$ and Enrique Font ${ }^{2}$ \\ ${ }^{1}$ Statistics Group, ARS, USDA, 10300 Baltimore Ave., Building 005, Beltsville, MD 20705, \\ USA (email: matt.kramer@ars.usda.gov) \\ ${ }^{2}$ Laboratorio de Etología, Instituto Cavanilles de Biodiversidad y Biología Evolutiva, \\ Universidad de Valencia, c/ Catedrático José Beltrán 2, 46980 Paterna, Valencia, Spain (email: \\ enrique.font@uv.es)
}

\begin{abstract}
Reducing the number of animal subjects used in biomedical experiments is desirable for both ethical and practical reasons. Previous suggestions for reducing sample sizes in these experiments have focused on improving experimental designs and methods of statistical analysis; reducing the number of controls (thus, the number of overall animals used) is rarely mentioned. We discuss how the number of current control animals can be reduced, without loss of statistical power, by incorporating information from historical controls, i.e. animals used as controls in similar previous experiments. Using example data from the literature, we describe how to incorporate information from historical controls under a range of assumptions, implemented either as familiar $t$-tests, contrasts, or in a mixed models context. Assuming more similarities between historical and current controls yields higher savings and allows the use of smaller current control groups. We conducted simulations, based on typical designs and sample sizes, to quantify how different assumptions about historical controls affect the power of statistical tests. Under our simulation conditions, the number of current control subjects can be reduced by more than half by including historical controls in the analyses. Paying attention to both the function and to the statistical requirements of control groups would result in reducing the total number of animals used in experiments, saving time, effort and money, and bringing research using animals within ethically acceptable bounds.
\end{abstract}

Key words: animal testing, animal welfare, borrowing information, control group, reduction, sample size, statistical power, three Rs.

\section{Introduction}

Although precise figures are lacking, a great majority of basic and pre-clinical biomedical research is conducted using laboratory animals, particularly mice and rats. Research with animals must comply with ethical guidelines that require, among other things, that researchers use the minimal number of animals necessary to achieve a scientific goal. Reducing the number of animals used in experiments is desirable for both ethical and practical reasons but must be based on a rational optimization process (i.e. pilot studies, formal power analyses) rather than tradition or prevailing constraints. One way to reduce the number of animals in an experiment is to reduce the size of current control groups. Here we demonstrate several strategies for incorporating information from historical controls, and, by so doing, reduce the number of current controls needed. Historical controls have been considered an undesirable option (Altman \& Bland, 1999; 
Keenan et al., 2009; Neuenschwander et al., 2010), particularly when the historical information is obtained from sources external to the laboratory conducting the research (Food and Drug Administration, 2001). This is because known or unknown biases can differentially affect subjects in the various control groups when all subjects are not drawn contemporaneously from the same population. However, we show that, judiciously used (and the keyword here is 'judiciously', as explained below), historical controls improve the researcher's ability to detect treatment effects and provide a useful tool for reducing the number of animals used as controls.

The focus of this paper is to provide a framework, largely in the mixed-models paradigm, to reduce the number of control animals needed in biomedical experiments without compromising the ability to detect a statistically significant treatment effect. We believe that the approaches and arguments outlined here will provide a valuable aid for researchers and contribute to alleviating the ethical burden of performing experiments with animals.

\section{Controls}

Researchers may be uncertain about what the right controls are for a given experiment or whether they are really needed. This is not surprising as conventional statistics textbooks used by biologists typically have little discussion on controls, neither their purposes nor how to integrate them into the experimental design. When mentioned, the advice is to consider them as another treatment group. Unfortunately, neither researchers nor their consulting statisticians receive adequate training on how best to incorporate controls into experiments, often each believing that the other has the expertise.

The various types of controls used in experiments are explained clearly in Johnson \& Besselsen (2002). They categorize controls as positive, negative, sham, vehicle (to test, e.g. the delivery system of a drug by itself), and comparative (positive control with a known treatment). In a negative control, control subjects remain in the 'normal' pre-experimental state; no change is expected from the pre-experimental to experimental condition. In a positive control, subjects receive some kind of pre-treatment (e.g. a toxin, a lesion) that is expected to cause a change from the pre-experimental state. The researcher can then see if a treatment (e.g. a vaccine) is effective by comparing treated subjects with subjects that do not receive a treatment. The positive control guards against miraculous recoveries, the negative against spontaneous disease and death. Sham controls are subjected to a manipulation that mimics the procedure received by positive controls and treated animals, but nothing else. Placebo controls often are considered negative controls in some clinical trials, but are more correctly a type of sham control. In fact, labeling controls as 'positive', 'negative', etc., may not provide much clarity. Often a short explanation is better than a label. It is important to understand what the purpose of the control is and whether it is the right kind of control for the experiment; some experiments may require several kinds of controls.

How many control subjects are needed in an experiment? This should depend on knowledge gained from the results of prior experiments. The cumulative knowledge in the field provides historical information that can be put to good use to reduce the number of animals in current control groups. The Bayesian statistical framework formally includes prior information when estimating a statistical model (e.g. French, Thomas \& Wang, 2012). However, biomedical research typically does not take advantage of this: most researchers conducting biomedical experiments on animals continue to use 'classical' Fisherian statistics (Efron, 2013), and there is little published guidance on how to incorporate information from prior experiments (Pocock, 1976; Neuenschwander et al., 2010; Viele et al., 2014). Since controls are included in most experiments, in the typical experiment we actually know far more about the control group than 
about the treatment group, either through experience with controls in similar experiments or through the literature (Schulz \& Grimes, 2005). However, we ignore this prior knowledge when we analyze the results of an experiment carrying out a classical statistical analysis, like a $t$-test or analysis of variance (ANOVA) using only animals involved in the current experiment. A laboratory performing similar experiments over many years will be far more sensitive to controls responding in unanticipated ways than to peculiar responses of treatment groups. Why not incorporate this prior knowledge?

Controls can be current or historical. Current or concurrent controls are contemporaneous with the treatment group(s), whereas historical, retrospective or background controls are obtained from prior experiments where similar protocols were applied to controls. Although the use of historical controls has been widely discussed in the literature, many researchers are unaware of their potential usefulness or do not know how to incorporate them into their experimental designs. In fact, the use of historical controls in biomedical research appears restricted to some areas of toxicology and to certain kinds of clinical trials involving humans. In these types of research, the dependent variable is usually a proportion (dichotomous or binary outcomes), and historical controls may not come from the same laboratory, potentially introducing an additional source of variation, often termed 'laboratory bias'.

A basic concern about using these historical controls is whether it is valid to test if the proportion from a current treated group differs from that of a historical control. The answer depends on whether historical controls provide a good estimate for what one would have obtained had one used current controls, and how one handles the data statistically. These various possibilities have been laid out in a Bayesian context by Spiegelhalter, Abrams \& Myles (2004) and Viele et al. (2014); they range from ignoring current controls (i.e. use only information from historical controls) to ignoring historical controls (traditional analysis). For quantitative data where normal distribution theory can be applied, there are additional options for using historical data, as discussed below. Thus, a basic difference between the previously suggested use of historic controls in clinical trials (using humans) and our suggested use for biomedical experiments (using animals) is that historical controls in clinical trials typically come from different laboratories, where critics can, with some justification, complain about difficult to quantify laboratory biases. Instead, we advocate borrowing historical control information only when there is a reasonable expectation of no laboratory biases (see below).

Historical controls can be used to better estimate parameters related to the current experiment under a variety of assumptions. Under the strongest assumption, if one has a large number of historical controls and one assumes that they are stable, i.e. neither the mean nor the variance of the historical controls change over experiments, then one can consider the historical control mean and variance to be fixed and only the current control and treatment groups have sampling error uncertainty. In this case, the need for a current control is debatable (other than to monitor laboratory conditions or to increase the number of historical controls available for future experiments). One has only to decide whether the treatment group mean and variance need to be estimated or just the mean, i.e. assume that the historical control groups provide a better estimate of the true within-group variance. This essentially is the framework used in the literature for conditional tests (conditional on the control group parameters considered as constants); the interest here is in differences of rates (proportions) (e.g. Yanagawa \& Hoel, 1985; Yoshimura \& Matsumoto, 1994). This use of historical controls can result in substantial reduction in experimental animal use. According to Browne (1976), for a given power and significance level, the estimated sample sizes are between one-quarter and one-half those needed if no historical controls are used (but see Lee \& Tseng, 2001). 
A less stringent assumption holds that there is only sampling variability in both historical and current controls, and that all controls vary about the same mean. In that case historical and current control data can be pooled, i.e. these observations are exchangeable, giving a larger sample size to estimate the control mean. If this model is not considered appropriate, models with fewer assumptions can be used, e.g. to allow for random experiment-to-experiment variation by including a random effect for experiments, or for treatments and controls to have different variances; in these cases the observations are not exchangeable. The analysis is then done in the mixed-models framework, which makes it possible to 'borrow' information from previous experiments on both means and variances. This is a kind of 'dynamic' borrowing of information, the amount of borrowing dictated by the quantity and characteristics of historical control data (Viele et al., 2014). A related approach, that we do not consider further, is subjectively discounting but not completely ignoring historical control data, as explained in Spiegelhalter et al. (2004).

A further relaxing of assumptions entails use of historical controls only to estimate variances since larger sample sizes are needed to estimate variances than means, and to use the current control group only for estimating the control mean, i.e. borrowing information across experiments only for variance estimation. This will reduce the number of animals compared to using data only from the current experiment but not as much as for the stronger assumptions discussed above. Here the gain one obtains is largely due to the increased residual degrees of freedom involved in the contrast between the current control group and the treatment groups.

In this paper we first describe how to incorporate (i.e. borrow) information from historical controls under different sets of assumptions using data from a series of nutrition experiments with rats. We then use simulations to demonstrate potential savings from using historical controls and to illustrate a few other points. We also provide suggestions about the use of historical controls for quantitative and proportion data.

\section{Experimental data}

Our exemplary data are haemoglobin concentrations $(\mathrm{g} / 100 \mathrm{ml})$, measured using an electronic cell counter, of Sprague-Dawley rats in a series of copper-deficiency experiments run over several years (Fig. 1; data from Reeves \& DeMars, 2004; Reeves et al., 2005; Saari et al., 2006; Relling et al., 2007; Johnson \& Johnson, 2009). There were eight groups of control rats available for our analysis, with various numbers of individuals per group. As can be seen in Fig. 1, means of these controls varied among the groups (13.3-15.0); we chose the control group with the highest mean to act as the current 'treatment' group (G.t in Fig. 1) and one of the two groups with five observations to act as the current 'control' group (G.c in Fig. 1). While every data set is unique, these data were chosen because they appear to be fairly typical of control measurements collected over several years on a laboratory animal, thus are 'real data', even if group labeling is contrived. 


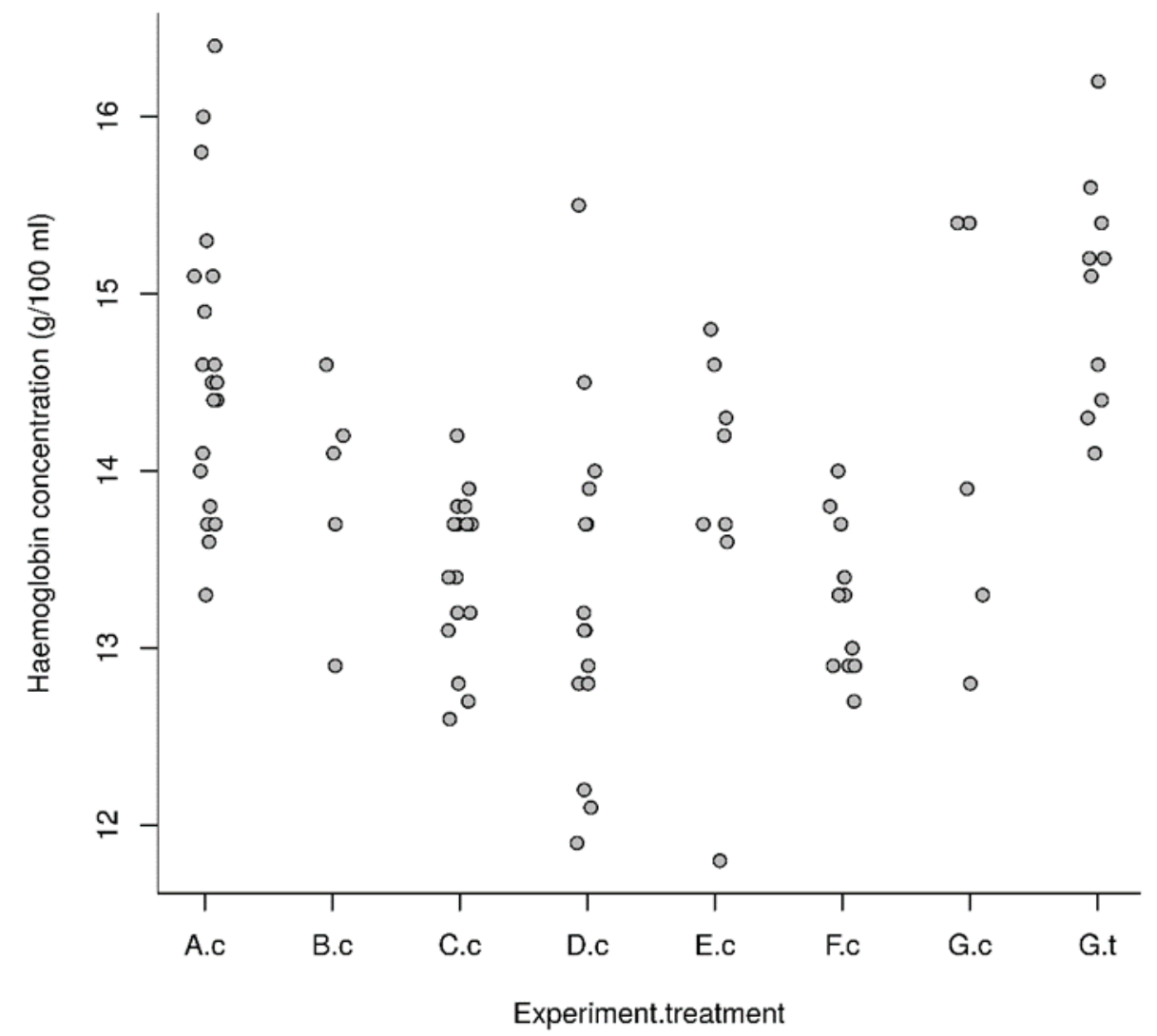

Figure 1. Haemoglobin concentrations $(\mathrm{g} / 100 \mathrm{ml})$ for control rats in eight nutrition experiments used to illustrate how different assumptions about incorporating historical controls in statistical testing affects outcomes. The historical controls are named A.c - F.c. In the current experiment, G.c is the control group and G.t the treatment group (rats in the G.t group were actually controls in the original experiment, but had the highest mean among the control groups, so we use them as the current 'treatment' group for illustrative purposes).

We tested for a difference between a treatment group and controls using a variety of statistical models with assumptions corresponding to those outlined above (Table 1; see Table 2 for underlying statistical models and Fig. 2 for illustrative examples). We abbreviate "assumption set" as "AS" here and elsewhere in this paper. These AS's determine how much information we can borrow from historical controls, that is, how much we allow parameter estimates from historical controls to influence parameter estimates from current controls. Models, in decreasing order of borrowing information, are as follows.

(AS 1) Many historical controls are available and we can consider controls to have a fixed mean; use a one-sample $t$-test to determine if the treatment mean differs from a constant (the control mean); our exemplary data are not consistent with these assumptions.

(AS 2) Assume that controls are stable and pool current and historical controls; use a two-sample $t$-test or ANOVA to test if the treatment group mean differs from that of the pooled control mean; 
exemplary data are not consistent with these assumptions.

(AS 3) Assume that historical controls are relatively stable but allow for an experiment-toexperiment random effect, i.e. the current control and treatment group comprise one block; fit a linear mixed model, with experiment as a random effect, and two levels of the treatment factor (treated versus control), this contrasts the adjusted treatment mean to the overall adjusted control mean; exemplary data are consistent with these assumptions.

(AS 4) Same as (AS 3) but allow the treatment group to have a different within-group variance than control groups; fit a linear mixed model as above, with control groups all sharing one within-group variance and estimate a different within-treatment group variance; this model is over-parameterized for our exemplary data.

(AS 5) Assume that historical control means are not sufficiently stable to use for comparison, and within-control group variances are stable but they differ from the treatment variance, allow for an experiment-to-experiment random effect; use the same linear mixed model as (AS 4) but create a 1 d.f. contrast between the current treatment and control groups; over-parameterized for our exemplary data.

(AS 6) Same as (AS 5) except model as a typical ANOVA so all groups share a common withingroup variance; fit a linear model with each group as a factor level, create a 1 d.f. contrast between the current treatment and current control groups; over-parameterized for exemplary data. (AS 7) Assume that historical control groups are not useful; test only current controls against the treatment group using a $t$-test or ANOVA. This is the typical assumption in most research laboratories where potentially useful information from historical controls is ignored; exemplary historical data ignored.

Results under the various assumptions follow, in reverse order. AS 7: if only current groups are compared (G.c versus G.t), the $P$ value from an ANOVA (1, 13 d.f.) on an estimate of the difference between means of 0.85 (S.E. =0.47) is 0.094. AS 6 \& 5: if an ANOVA is applied to the whole data set and an a priori contrast between G.c and G.t made, with 82 d.f., the estimate is still 0.85 , but the S.E. is now 0.42 and the $P$ value 0.047 . We have borrowed information from other control groups, so have a better estimate of the 'true' S.E. for the contrast, and have more degrees of freedom to test it. The same results are obtained if we model the factor, experiment, as a random effect, with the experiment-to-experiment variance estimated to be 0.328. AS $4 \&$ 3: if we assume for these exemplary data that all control groups share an underlying true common mean but there is a random experiment-to-experiment effect, the treatment-control difference is estimated as 0.99 (S.E. $=0.37$ ) with a $P$ value of 0.010 ; we get similar results assuming unequal variances. AS 2: if we had simply pooled over controls, the treatment-control difference is 1.22 and, using ANOVA, the $P$ value is 0.0001 on 1 and 88 d.f. AS 1: if we test the treatment group against a constant control mean $(=13.79$ over all controls, difference with treatment group $=$ 1.22) using a $t$-test, on 9 d.f., the $P$ value $=0.0002$.

As stronger assumptions are made, $P$ values decrease and therefore, our ability to detect a statistically significant treatment effect increases. However, even for AS $6 \& 5$, there is a benefit to including the historical controls; fewer concurrent controls are needed to achieve the same power if historical controls are included. 
Table 1. Summary of assumption sets regarding historical controls.

\begin{tabular}{|c|c|c|c|c|c|c|c|}
\hline \multirow[t]{2}{*}{ Assumptions } & \multicolumn{7}{|c|}{ Assumption set } \\
\hline & 1 & 2 & 3 & 4 & 5 & 6 & 7 \\
\hline $\begin{array}{l}\text { Control } \\
\text { mean } \\
\text { considered } \\
\text { fixed }\end{array}$ & yes & no & no & no & no & no & no \\
\hline $\begin{array}{l}\text { Borrow } \\
\text { mean } \\
\text { information } \\
\text { from HC }\end{array}$ & NA & yes & yes & yes & no & no & no \\
\hline $\begin{array}{l}\text { Borrow } \\
\text { variance } \\
\text { information } \\
\text { from HC }\end{array}$ & no & yes & yes & yes & yes & yes & no \\
\hline $\begin{array}{l}\text { Experiment- } \\
\text { to- } \\
\text { experiment } \\
\text { random } \\
\text { effects }\end{array}$ & no & no & yes & yes & yes & no & no \\
\hline $\begin{array}{l}\text { Within- } \\
\text { group } \\
\text { variances }\end{array}$ & NA & $\begin{array}{l}\text { all the } \\
\text { same }\end{array}$ & $\begin{array}{l}\text { all the } \\
\text { same }\end{array}$ & $\begin{array}{c}\mathrm{T} \& \mathrm{C} \\
\text { can } \\
\text { differ }\end{array}$ & $\begin{array}{l}\mathrm{T} \& \mathrm{C} \\
\text { can } \\
\text { differ }\end{array}$ & $\begin{array}{l}\text { all the } \\
\text { same }\end{array}$ & $\begin{array}{l}\text { all the } \\
\text { same }\end{array}$ \\
\hline
\end{tabular}

See text for description of assumption sets.

$\mathrm{HC}$, historical control groups; NA, not applicable; T \& C, treatment and control groups. 
Table 2. Models and tests for assumption sets.

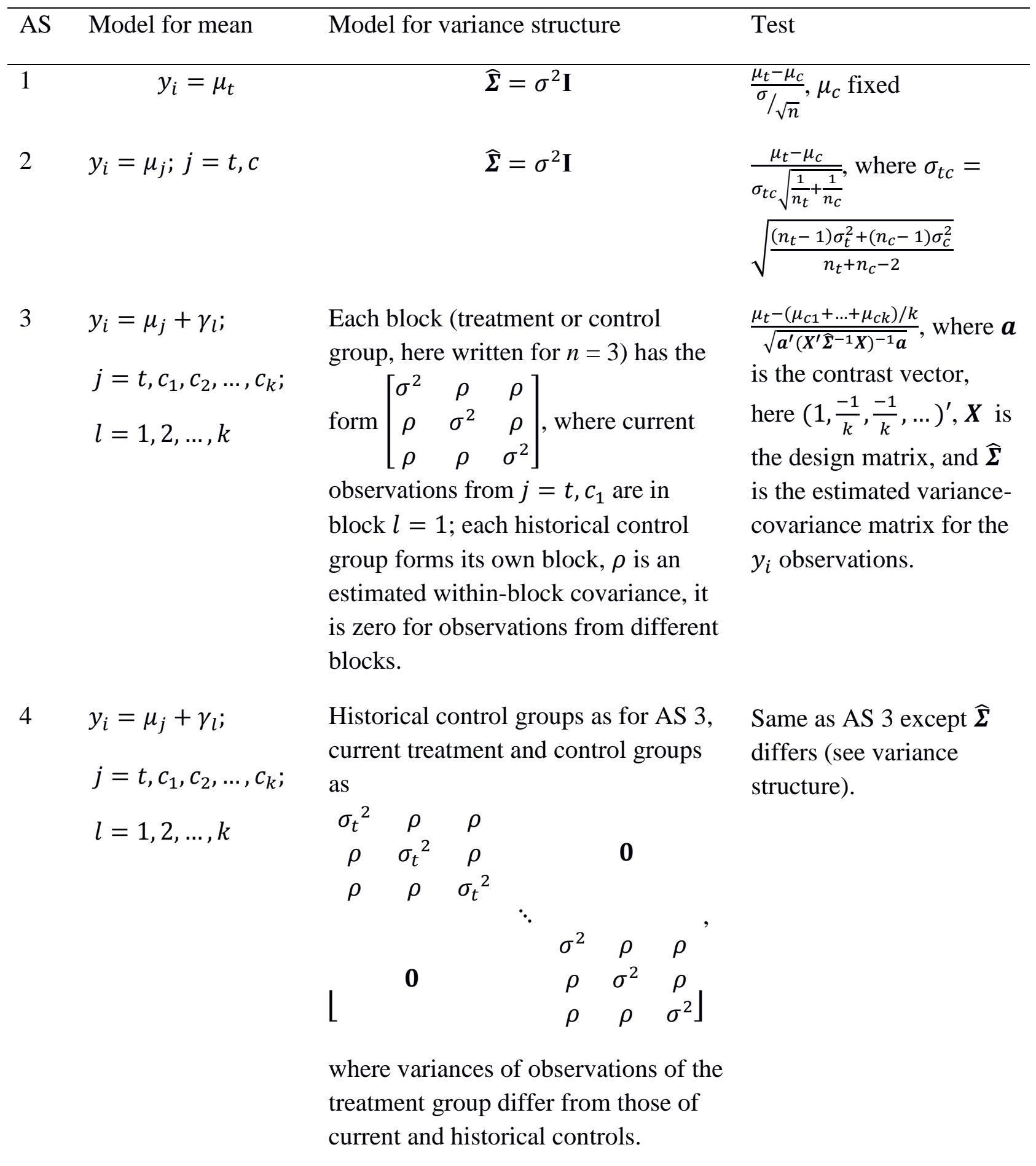



$5 \quad y_{i}=\mu_{j}+\gamma_{l}$
Same as AS 4
$j=t, c_{1}, c_{2}, \ldots, c_{k}$
$l=1,2, \ldots, k$
6

$$
\begin{aligned}
& y_{i}=\mu_{j} ; \\
& j=t, c_{1}, c_{2}, \ldots, c_{k}
\end{aligned}
$$
$\sigma^{2} \mathbf{I}$ differs (see variance structure).
Same as AS 5 except $\widehat{\boldsymbol{\Sigma}}$
$7 \quad y_{i}=\mu_{j} ; j=t, c_{1}$
$\sigma^{2} \mathbf{I}$
Same as AS 2 but only is used. the current control group

\begin{abstract}
These are not meant to be complete descriptions since the expressions are mostly written out for equal sample sizes to avoid including weightings, but should be sufficient to understand how models for the assumption sets (see Table 1) differ. Also, to avoid making the notation more complicated and to connect better to the other columns, the expressions in the test column are given using parameters rather than estimates of the parameters (i.e. $\sigma$ [population standard deviation] rather than $s$ [sample standard deviation], $\mu$ [population mean] rather than $\bar{X}$ [sample mean]). The random block (group) effect is notated as $\gamma$, with the number of observations in a group represented by $n$. The subscript $i$ indexes observations $(y), j$ indexes means of treatment $\left(\mu_{t}\right)$ or control groups $\left(\mu_{c 1}, \mu_{c 2}, \ldots\right)$, and there are $k$ blocks (groups), indexed with $l$. The symbol I represents the identity matrix, the symbol ' indicates the matrix transpose operation. Other symbols are defined in the table. Complete formulae can be found in statistics books covering linear mixed models, e.g. Milliken \& Johnson (2009).
\end{abstract}

\title{
4. Simulations
}

To quantify how the various assumptions affect statistical test results, we ran simulated data sets $(N=5000)$ testing for differences between controls and the treatment group through standard statistical models in R [ $1 \mathrm{~m}$ and t.test in base R (R Core Team, 2013), lme in the nlme package (Pinheiro et al., 2013)]. Example R code is given in the Appendix. We picked a set of characteristics that we felt would be typical for biomedical studies involving animals, and similar to those from our exemplary data in the extent to which control group means differed from each other. We used the same variance for all groups; models allowing for different variances are overparameterized. Thus, AS 3 to AS 7 are satisfied since the simulated data were generated with varying control group means but the same variance, while AS 1 and 2 are not, since they assume that all control group means and variances are the same. However in AS 1, the control is considered fixed; the only control information used in the test is a fixed mean (and we used the true mean), so simulation results are not affected by the violation. AS 2 is not met because control group means are affected by introduced experiment-to-experiment variation. We do not give simulation results from AS 2; power for two-sample $t$-tests can easily be obtained using available software. 

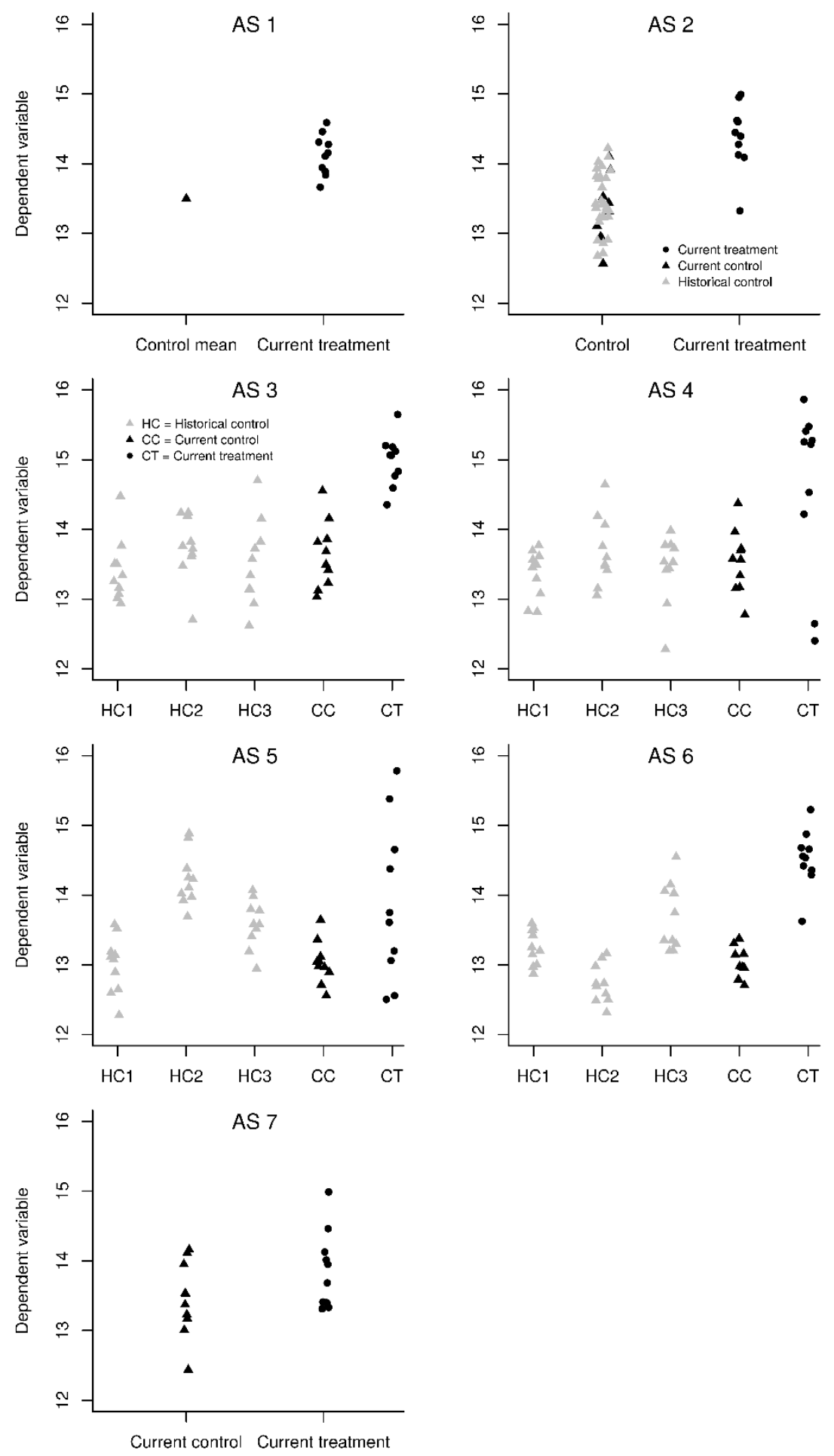

Figure 2. Illustrations of underlying assumptions for statistical models discussed in the text and detailed in Table 2 (data simulated). In all models, samples are drawn from a normal distribution. In assumption set 1 (AS 1), the control group estimate is considered fixed and measured without error. In AS 2, AS 3, AS 6, and AS 7, all groups have the same variance; in AS 4 and AS 5 the treatment group has a larger variance than control groups. 
Table 3. Simulation results $(N=5000)$ giving power to detect a difference between treatment and control groups under various assumptions. In this scenario, data were simulated from a series of related experiments, one current and others historical, as summarized in the first four columns.

\begin{tabular}{|c|c|c|c|c|c|c|c|c|c|}
\hline $\begin{array}{c}\text { Number of } \\
\text { current } \\
\text { controls }\end{array}$ & $\begin{array}{c}\text { Number of } \\
\text { current } \\
\text { treated }\end{array}$ & $\begin{array}{c}\text { Number of } \\
\text { historical } \\
\text { controls } \\
\text { per group }\end{array}$ & $\begin{array}{c}\text { Number of } \\
\text { historical } \\
\text { control } \\
\text { groups }\end{array}$ & $\begin{array}{l}\text { Power for } \\
\text { a fixed } \\
\text { control } \\
\text { mean } \\
\text { (AS 1) }\end{array}$ & $\begin{array}{l}\text { Power } \\
\text { assuming } \\
\text { stable } \\
\text { control } \\
\text { means and } \\
\text { equal } \\
\text { variances } \\
\text { (AS 3) }\end{array}$ & $\begin{array}{l}\text { Power } \\
\text { assuming } \\
\text { stable } \\
\text { control } \\
\text { means and } \\
\text { unequal } \\
\text { variances } \\
\text { (AS 4) }\end{array}$ & $\begin{array}{l}\text { Power } \\
\text { assuming } \\
\text { unstable } \\
\text { control } \\
\text { means and } \\
\text { unequal } \\
\text { variances } \\
\text { (AS 5) }\end{array}$ & $\begin{array}{l}\text { Power } \\
\text { assuming } \\
\text { unstable } \\
\text { control } \\
\text { means and } \\
\text { equal } \\
\text { variances } \\
\text { (AS 6) }\end{array}$ & $\begin{array}{c}\text { Power } \\
\text { ignoring } \\
\text { historical } \\
\text { controls } \\
\text { (AS 7) }\end{array}$ \\
\hline 3 & 5 & 5 & 4 & 0.62 & 0.65 & 0.65 & 0.42 & 0.42 & 0.34 \\
\hline 3 & 5 & 5 & 3 & 0.61 & 0.64 & 0.62 & 0.41 & 0.41 & 0.34 \\
\hline 3 & 5 & 5 & 2 & 0.61 & 0.60 & 0.61 & 0.40 & 0.40 & 0.34 \\
\hline 3 & 5 & 5 & 1 & 0.62 & 0.55 & 0.54 & 0.39 & 0.39 & 0.34 \\
\hline 6 & 5 & 5 & 3 & 0.61 & 0.67 & 0.67 & 0.56 & 0.55 & 0.50 \\
\hline 9 & 5 & 5 & 3 & 0.61 & 0.71 & 0.71 & 0.63 & 0.63 & 0.59 \\
\hline 12 & 5 & 5 & 3 & 0.61 & 0.73 & 0.72 & 0.67 & 0.67 & 0.65 \\
\hline 15 & 5 & 5 & 3 & 0.61 & 0.75 & 0.75 & 0.71 & 0.71 & 0.69 \\
\hline 3 & 10 & 5 & 3 & 0.96 & 0.80 & 0.79 & 0.49 & 0.49 & 0.44 \\
\hline 3 & 15 & 5 & 3 & 1.00 & 0.86 & 0.86 & 0.53 & 0.53 & 0.50 \\
\hline 4 & 5 & 5 & 3 & 0.60 & 0.65 & 0.64 & 0.47 & 0.47 & 0.40 \\
\hline 3 & 5 & 3 & 3 & 0.61 & 0.59 & 0.59 & 0.40 & 0.40 & 0.35 \\
\hline
\end{tabular}

The true within-group S.D. was 3 for all groups. For all simulated data sets, historical control means were samples from a normal distribution (mean $=10$, S.D. $=1$ ), and the current control mean $=10$, the current treatment mean $=$ 14. Models for columns 5-10 are as follows (AS = assumption set): column 5, test against a fixed control mean of 10 (AS 1); column 6, random experiment effect (i.e. experiment 1 had current controls and treated, experiment 2 had one group of historical controls, experiment 3 had one group of historical controls, etc.), within-group variances all assumed equal, test (adjusted) current treated mean against the (adjusted) mean of all controls (AS 3); column 7 , same as column 6 except variance of treated group estimated separately (AS 4); column 8, same as column 7 except test current treated against the current control as a contrast, with historical data included in parameter estimation (AS 5); column 9, same as column 8 except using ANOVA (no random effects, equal variances) (AS 6); column 10, test current treated against current control (AS 7). 
The simulations were set up with all groups having the same S.D. of 3, but with group means differing. Means of historical controls were samples drawn from a normal distribution with overall mean 10, S.D. = 1, current control group with mean 10, current treatment group with mean 14 (effect size, Cohen's $d=4 / 3$ ). We varied the sample sizes of the current treatment and control groups, the number of historical control groups included when testing, and the model assumptions as described above (see Table 1). Power was near 50\%, i.e. significance at $\alpha=0.05$ was detected for about half the simulated data sets, which allowed us easily to see effects of changing assumptions. We also ran the same set of simulations, but setting the current treatment mean to 10 , the same as the control means, to see if the nominal 5\% Type I error rate was respected by the software when making tests. We do not give results for this. For the most part, as expected, approximately $5 \%$ of tests were significant, the exception being tests made under AS 4 above, where values ranged from $6 \%$ to $9 \%$. These liberal values suggest to us that variances are being slightly under-estimated by the software. This does not affect the conclusions drawn from the simulation results that we do report.

We provide results from the simulations in Table 3 . While these results apply directly only to the simulated data sets we created, the main conclusions drawn should be generalizable to other data sets with similar characteristics, like our exemplary data. Increasing the number of total subjects in control groups beyond 18 does little to improve power (first four rows, with four, three, two and one historical control groups) under any of the assumptions. Considering historical controls fixed (at mean $=10$, AS 1) always had high power (column 5), and was surpassed only when there were many residual degrees of freedom involved in the statistical tests due to having large numbers of historical control subjects available [columns 6 and 7 (AS 3 and 4)]. Allowing for unequal variances had little effect on power [column 6 versus 7 (AS 3 and 4), and column 8 versus 9 (AS 5 and 6); note that the true variances of all groups were the same, so the small differences seen are due only to the extra parameter estimated]. Large gains in power can be achieved by using historical controls; including historical controls always increased power unless a large number of current controls was used, compare column 10 (AS 7) with columns 6-9 (AS 3-6), for rows 2 and 5-8. Using current controls only [column 10 (AS 7), the usual situation in most experiments] had consistently the lowest power, followed by borrowing information only for variance estimation [columns 8 and 9 (AS 5 and 6)]. If one can assume that historical controls are relatively stable, as could be done for these simulated data, tests had high power [columns 6 and 7 (AS 3 and 4)]. Increasing the number of current controls (rows 5-8) had the largest impact on tests with the weakest assumptions [columns 8-10 (AS 5-6)]. Increasing the sample size of the treatment group (rows 9-10) had its largest effect when testing against a fixed control mean [column 5 (AS 1)].

As an example of how many animals could be eliminated by designing an experiment using historical controls under the assumption that control means are stable (AS 3 and 4), using three current controls and 20 historical controls yielded the same power (0.65) as using 12 current and no historical controls, thus one could eliminate nine animals from the current experiment (i.e. use a total of eight animals in the current experiment instead of 17) without sacrificing any power. Fewer current controls could be eliminated if one only borrows variance information from historical controls, but nonetheless some savings are possible. 


\section{When can historical controls be used?}

Biomedical experiments are centered on a narrow range of model organisms, often in highly controlled settings. In many research laboratories, an experiment is not an isolated event but one of a sequence of similar experiments using similar procedures and experimental animals. If treatments vary but control conditions stay the same for long series of experiments, there should be no controversy using historical controls (Festing \& Altman, 2002). In less clear cases, one can rely on a combination of familiarity and expertise with the system, perhaps with a small pilot study, to make the decision. The key for designing an appropriate control group is that it is identical to the treatment group in every way except for the treatment being tested. To the extent that historical controls meet this requirement their use-in conjunction with current controls-will be justified. Generally, it should be safe to use historical controls from previous experiments conducted in the same laboratory using the same species and procedures (Greim et al., 2003; Keenan et al., 2009; Hayashi et al., 2011). This recommendation pertains to all the various types of controls.

Consistency between historical and current experimental conditions should be given paramount consideration in the decision to incorporate historical controls as part of the experimental design. If historical controls are inconsistent with current controls, there is a potential for bias and increased type I error. Drift in control values over time, e.g. cure rates affected by evolving antibiotic resistance, are a clear case where historical and current controls are not consistent. Lack of stability in controls may also reflect changes in study design-related parameters such as species/strain, eligibility criteria, route of administration, vehicle, reagents, chemical and equipment suppliers, feeding and housing practices, or may be the result of sampling error as when the measurements of interest have inherently high variance. Unexplained fluctuations in the frequency of spontaneous tumours in control rats have been reported in some research facilities (Ando et al., 2008; Kuroiwa et al., 2013). However, what little information is available suggests that, when laboratories are well managed, controls are repeatable. For example, Hayes et al. (2009) report that the data for 116 control groups (each group with $N=7$ rats) run over several years in the same laboratory were highly repeatable; the rats served as vehicle controls for the bone marrow micronucleus test. The authors conclude that 'no significant experimental variability was seen within or between control animals' (p. 423). This conclusion holds despite the fact that the source of the rats changed midway through these years of data collection. In this example, all control groups, regardless of origin, were maintained in the same laboratory. This differs from clinical studies in which historical controls are obtained from sources external to the laboratory conducting the research (Thomas, 2008). The latter use of historical controls is controversial because unaccounted differences between laboratories, researchers, patients or experimental protocols may render them unsuitable as a reference against which current treatment groups can be compared (Diehl \& Perry, 1986). Thus, even though it is generally agreed that designs using historical controls are highly desirable for ethical and economic reasons (e.g. Gehan \& Freireich, 1974; Cranberg, 1979), the evidence they provide is often considered weaker than, e.g. randomized clinical trials (Doll \& Peto, 1980; Pocock, 1983). Our proposed use of historical controls is more conservative; it is based on the use of control data from previous experiments conducted in the same laboratory rather than on external data sources. The decision whether to use historical controls entails a trade-off: the potential introduction of some bias in the current study if experimental conditions have changed (i.e. 'historical bias') versus the reduction of current controls allowed by adding historical information.

Unfortunately, there is no automatic, foolproof method to help the researcher decide 
whether or not to include historical controls when only one or two historical control groups are available. In fact, to make the determination based on statistics alone would require the large sample sizes we advocate eliminating. If many historical control groups are available, we recommend using statistical quality-control methods. These methods for determining the consistency of a process are well developed and can be used to monitor control animal performance over a series of experiments (for examples see Festing \& Altman, 2002; Hayes et $a l ., 2009)$; they may already be instituted in well-run laboratories. There are control methodologies for both means (X-bar charts) and standard deviations (S-charts) (Wheeler \& Chambers, 1992). We created standard quality-control charts for the eight control data sets of rat haemoglobin concentrations and found that three of the groups (A.c, D.c, G.t; see Fig. 1) exceeded the X-bar chart confidence limits (i.e. the assumption of a common mean is not supported). However, none exceeded the $\mathrm{S}$-chart confidence limits (i.e. assuming a common standard deviation or variance is reasonable). These data would have satisfied AS 6, and also AS 3 if the kind of variation seen in the means was typical experiment-to-experiment variability, i.e. could be modeled as a random effect, or had additional information explaining why these control groups differed, e.g. due to different age or sex compositions.

What assumptions are applicable in a particular case depends largely on the type of historical controls available. If the experimental protocol remains unchanged throughout the period when historical control data were collected and there is little variation among controls over time, one may assume that historical controls have a fixed mean (and variance) and use them to estimate parameters relating to the current experiment. With less-stable historical controls the researcher has to decide whether it makes sense to incorporate information from their mean and/or variance. Assuming that historical control groups can be used to estimate a common control mean and variance allows for a greater reduction in animals than assuming that controls can only be used to estimate a common variance.

Randomization and blinding are essential aspects of experimental design, but their application to historical controls is not straightforward. Blinding may be hard to implement but randomization should not be an issue in most cases. Subjects should be randomly allocated to current control and treatment groups, and suitably randomized in the experiments from which the historical controls are taken, but randomizing across experiments has been constrained, i.e. each experiment can be considered a constraint on randomization. This is the classic situation producing a randomized block design. The mixed models take that into account by considering each experiment as a block (see Table 2).

\section{Determining sample sizes when using historical controls}

Currently, there is no off-the-shelf software for determining how to substitute historical controls for current controls for many of our assumption sets. We suggest determining sample size using the usual tools for current controls only (AS 7), then determine how many historical controls can be substituted for each current control using simulations or 'rules of thumb', which will depend on which AS is used. For AS 2, each historical control is equivalent to one current control, i.e. there is a 1:1 substitution. AS 6 uses a contrast from a linear model, so sample sizes can be calculated using standard statistical software that estimates power, as long as contrasts can be specified. Based on our simulations for AS 3, that is, based on characteristics we dictated when creating data, substitution was conservatively 2:1 (historical:current). This can be seen in Table 3 by comparing columns 6 and 10 where power is approximately the same. In one case, the ratio is 10:6 (historical:current), in another it is 15:9 (this is after adjusting for the three current controls 
under AS 3). This result is only loosely generalizable: a different set of characteristics would yield different ratios. AS 3-6 are built on mixed models, where assessing power is not trivial since models can vary in many dimensions; simulation currently provides the most accurate estimate of power under different allocations of controls (Johnson et al., 2015). Software for calculating power and sample size for experiments like these is readily available for AS 1,2 , and 7. It can also be found for AS 3 (blocks may be labeled 'clusters'), for example, in the software MLPowSim (which can write R scripts or call MLwiN for estimation; downloadable from http://www.bristol.ac.uk/cmm/software/mlpowsim/), OD (hlm.soft.net/od/), and GLIMMPSE (glimpse.samplesizeshop.org, which uses the general linear multivariate model parameterization). Another on-line tool, SMEEACT (research.mdacc.tmc.edu/SmeeactWeb/Default.aspx) uses a Bayesian approach and can provide the weight that should be given to historical controls, based on their stability, for a set of anticipated or realized data. We do not know of software packages that can be used off-the-shelf for these purposes for AS 4, 5, 6 and 8; see Appendix for the R code.

We do not advocate borrowing information from historical controls for analyses using proportion data, e.g. proportion that improved after receiving a treatment, unless one can make the assumption that control proportions are constant over time. This is the basic strategy taken by Korn \& Freidlin (2006) and others dealing with proportion data (e.g. Yanagawa \& Hoel, 1985; Hayashi et al., 1989; Ryan, 1993; Yoshimura \& Matsumoto, 1994). The reason for this lies in the difference between the normal distribution and other members of the exponential family of distributions (e.g. binomial, Poisson). The normal distribution has the property that the mean and variance are independent. Because of this property, if one assumes that within-control group variances are relatively constant, it makes sense to estimate this variance from a large number of observations: by borrowing information from historical data we increase the number of observations. However, the binomial and Poisson distribution are one-parameter distributions, with a single parameter and the known sample size determining both the mean and variance. Unless the control proportion rate is stable, borrowing information from historical controls in a proportion data set which is assumed to be generated by a binomial process would not increase the precision of the current control proportion since there is no independent variance estimate. We should use historical control data to assess whether variability over time in controls is greater than that expected from sampling error alone (i.e. resulting in over-dispersion) as a means of monitoring stability in laboratory conditions. Models for binomial data that include historical control groups as random effects (Maringwa et al., 2007) can improve the estimate for the true response proportion of controls (this is in the generalized linear mixed-models framework). These models can also allow for over-dispersion, a common feature of binomial data, and the additional historical controls will improve the estimate of over-dispersion.

\section{Recommendations}

The three Rs of replacement, reduction and refinement originally proposed by Russell \& Burch (1959) provide a widely accepted framework for conducting animal experiments. One area where progress has been somewhat limited is in the reduction of the number of animals included in an experiment. Reduction entails seeking ways of obtaining comparable levels of information from the use of fewer experimental animals, or of obtaining more information from a given number of animals (Festing et al., 1998). Compared to refinement and replacement, which often require technical advances, implementing reduction strategies has an immediate impact on animal welfare. Reducing the number of animals will also result in a reduction of the resources and 
workload required to run an experiment. Therefore, for both ethical and economic reasons, experiments should be designed such that they use the minimum number of animals necessary to achieve meaningful scientific results. Reduction of the number of current controls provides a relatively straightforward means to this end.

We are not advocating that researchers eliminate or arbitrarily reduce control groups. Quite to the contrary, our aim here is to convince the reader that control groups deserve far greater attention than is current practice. A thorough understanding of the role of controls is crucial to conducting research that is both effective and ethically acceptable. Experiments in which controls are subjected to painful or stressful procedures with a highly predictable endpoint are relatively common and should be given special consideration. Recent surveys have shown that much animal experimentation is grossly underpowered (e.g. Button et al., 2013), and as a consequence a typical researcher may be hesitant to reduce the number of subjects used in an experiment. But if the aim of the controls is simply to guarantee that experimental procedures work adequately, a reduction may be fully justified on ethical grounds.

Under conditions discussed above, the power loss resulting from a reduction in the number of current controls can be offset by borrowing information from historical controls. This allows the researcher to minimize the amount of suffering while at the same time preserving statistical power. Our simulations show that there is always a benefit to including historical controls as fewer current controls are needed to achieve the desired power. Interestingly, the largest gains in power (up to 50\%) in our simulations are obtained when the number of animals in the treatment group is increased and historical controls are used. Thus, a researcher that has discretion over the number of subjects allocated to different groups may achieve a more powerful design by incorporating historical controls and relocating subjects from the current control to the treatment group.

The current discussion of the use of historical controls is targeted to biomedical experimentation, which arguably accounts for the largest number of procedures involving animals. However, the focus can be fruitfully broadened to other areas of research using animals. Circumstances will likely vary among different disciplines but drawing on historical control data will generally allow a reduction of the number of animals allocated to current control groups and therefore contribute to the goal of saving time, resources and animal lives. Additional details and a discussion of other aspects of the use of controls in biomedical experiments is given in Kramer and Font (2017)

\section{Summary}

Control groups are included in most experiments, and they offer a relatively untapped potential to reduce sample size without sacrificing statistical power. Controls can be categorized as current or historical. Historical controls are controls from past experiments that used the same protocols as the current experiment. Historical information is used to design most experiments (i.e. to calculate sample sizes based on previously observed variabilities and effect sizes). However, use of historical controls is restricted to a few areas of biomedical research and is regarded with skepticism by many researchers who are unaware of their potential usefulness or do not know how to incorporate them into their experimental designs. We show, using both a real data example and computer simulations, how to use historical controls under various sets of assumptions. In general, use of historical controls reduces the number of current controls necessary in an experiment and improves the researcher's ability to detect treatment effects. Borrowing information from historical controls entails a tradeoff between the potential 
introduction of bias (if historical controls do not adequately reflect current experimental conditions) and the reduction of current control subjects. Consistency between historical and current experimental conditions should be the most important consideration in the decision to incorporate historical controls as part of the experimental design. When similar experiments are performed repeatedly in the same laboratory, using the same standard research and husbandry protocols and the same animals (e.g. a single strain and sex of mice), there will often be scope for the incorporation of historical controls. Understanding the role of controls is crucial to conducting research that is both effective and ethically acceptable. An efficient use of controls can reduce the number of animals required and maximize the information obtained per experiment. Given the sheer number of animals that are routinely used as part of control groups, the procedures we outline can make a substantial contribution towards the goal of reducing the number of animals used in biomedical research.

\section{ACKNOWLEDGEMENTS}

We thank Gordon Burghardt, Pau Carazo, Marian Dawkins, Hal Herzog, Benjamin Rosenthal, Sonja von Aulock, Paul Weldon, and several anonymous reviewers for providing critical feedback on earlier versions of the manuscript. We thank LuAnn Johnson (ARS statistician, Northern Plains Area), who graciously assembled the control data used in our example, and Philip G. Reeves (deceased), W. Thomas Johnson and David P. Relling for agreeing to our use of their data.

\section{REFERENCES}

Altman, D.G. \& Bland, J.M. 1999. Statistics notes. Treatment allocation in controlled trials: why randomise? British Medical Journal 318, 1209.

Ando, R., Nakamura, A., Nagatani, M., Yamakawa, S., OHIRA, T., TAKagi, M., Matsushima, K., Aoki, A., Fujita, Y. \& TAmura, K. (2008). Comparison of past and recent historical control data in relation to spontaneous tumors during carcinogenicity testing in Fischer 344 rats. Journal of Toxicologic Pathology 21, 53-60.

BROWNE, R. H. (1976). Reducing sample sizes when comparing experimental and control groups. Archive of Environmental Health 31, 169-170.

Button, K. S., IoAnnidis, J. P. A., Mokrysz, C., Nosek, B. A., Flint, J., Robinson, E. S. J. \& MunAfÒ, M. R. (2013). Power failure: Why small sample size undermines the reliability of neuroscience. Nature Reviews Neuroscience 14, 365-376.

CRANBERG, L. (1979). Do retrospective controls make clinical trials "inherently fallacious"? British Medical Journal 2, 1265-1266.

DIEHL, L. F. \& PERRY, D. J. (1986). A comparison of randomized concurrent control groups with matched historical control groups: Are historical controls valid? Journal of Clinical Oncology 4, 1114-1120.

DOLL, R. \& PETO, R. (1980). Randomized controlled trials and retrospective controls. British Medical Journal 280, 44.

EFRON, B. (2013). Bayes' theorem in the 21st century. Science 340, 1177-1178. 
Festing, M. F. W. \& Altman, D. G. (2002). Guidelines for the design and statistical analysis of experiments using laboratory animals. ILAR Journal 43, 244-257.

Festing, M. F. W., Baumans, V., Combes, R. D., Halder, M., Hendriksen, C. F. M., Howard, B. R., Lovell, D. P., Moore, G. J., Overend, P. \& Wilson, M. S. (1998). Reducing the use of laboratory animals in biomedical research: Problems and possible solutions. The report and recommendations of ECVAM workshop 29. Alternatives to Laboratory Animals 26, 283-301.

FoOD AND DRUG AdMINISTRATION. Guidance for Industry: E 10 Choice of Control Group and Related Issues in Clinical Trials. Available at:

http://www.fda.gov/downloads/drugs/guidancecomplianceregulatoryinformation/guidances/ucm0 73139.pdf. Accessed August 5, 2015.

French, J.L., ThOMAS, N. \& WANG, C. (2012). Using historical data with Bayesian methods in early clinical trial monitoring. Statistics in Biopharmaceutical Research 4, 384-394.

GeHAN, E. A. \& FreIREICH, E. J. (1974). Non-randomized controls in cancer clinical trials. New England Journal of Medicine 290, 198-203.

Greim, H., GelbKe, H. P., Reuter, U., Thielmann, H. W. \& EdLER, L. (2003). Evaluation of historical control data in carcinogenicity studies. Human \& Experimental Toxicology 22, 541549.

Hayashi, M., Yoshimura, I., Sofuni, T. \& Ishidate, M. (1989). A procedure for data analysis of the rodent micronucleus test involving a historical control. Environmental and Molecular Mutagenesis 13, 347-356.

Hayashi, M., Dearfield, K., Kasper, P., Lovell, D., Martus, H.-J. \& Thybaud, V. (2011). Compilation and use of genetic toxicity historical control data. Mutation Research 723, 87-90.

Hayes, J., Doherty, A. T., AdKins, D. J., Oldman, K. \& O’Donovan, M. R. (2009). The rat bone marrow micronucleus test — study design and statistical power. Mutagenesis 24, 419-424.

Johnson, P. D. \& Besselsen, D. G. (2002). Practical aspects of experimental design in animal research. ILAR Journal 43, 202-206.

Johnson, W. T. \& Johnson, L. K. (2009). Copper deficiency inhibits Ca2+-induced swelling in rat cardiac mitochondria. Journal of Nutritional Biochemistry 20, 248-253.

Johnson, P. C. D., BArry, S. J. E., Ferguson, H. M. \& Müller, P. (2015). Power analysis for generalized linear mixed models in ecology and evolution. Methods in Ecology \& Evolution 6 , 133-142.

Keenan, C., Elmore, S., Carroll-Francke, S., Kemp, R., Kerlin, R., Peddada, S., Pletcher, J., RinKe, M., SCHMIDT, S. P., TAYLOR, I. \& WOLF, D. C. (2009). Best practices for use of historical control data of proliferative rodent lesions. Toxicologic Pathology 37, 679-693.

KORN, E. L. \& FREIDLIN, B. (2006). Conditional power calculations for clinical trials with historical controls. Statististics in Medicine 25, 2922-2931.

KRAMER M. \& FONT E.(2017). Reducing sample size in experiments with animals: historical controls and related strategies. Biol. Rev. 92, 431-445. 
Kuroiwa, Y., Ando, R., Kasahara, K., Nagatani, M., Yamakawa, S. \& OKaZaki, S. (2013). Transition of historical control data for high incidence tumors in F344 rats. Journal of Toxicologic Pathology 26, 227-230.

LEE, J. J. \& TSENG, C.-H. (2001). Uniform power method for sample size calculation in historical control studies with binary response. Controlled Clinical Trials 22, 390-400.

Maringwa, J. T., Faes, C., Aerts, M., Geys, H., Teuns, G., Poel, B. V. D. \& Bijnens, L. (2007). On the use of historical control data in pre-clinical safety studies. Journal of Biopharmaceutical Statistics 17, 493-509.

Neuenschwander, B., Capkun-Niggli, G., Branson, M. \& Spiegelhalter, D. J. (2010). Summarizing historical information on controls in clinical trials. Clinical Trials 7, 5-18.

Pinheiro, J., BAtes, D., DebRoy, S., SARKAR, D. \& R Development Core TEAm (2013). nlme: Linear and Nonlinear Mixed Effects Models. R package version 3.1-109.

PocOCK, S. J. (1976). The combination of randomized and historical controls in clinical trials. Journal of Chronic Diseases 29, 175-188.

Pocock, S. J. (1983). Clinical Trials - A Practical Approach. John Wiley \& Sons, Chichester.

PuOPOLO, M. (2004). Biostatistical approaches to reducing the number of animals used in biomedical research. Annali dell'Istituto Superiore di Sanità 40, 157-163.

R CORE TEAM (2013). R: A language and environment for statistical computing. R Foundation for Statistical Computing, Vienna, Austria ISBN 3-900051-07-0, URL http://wwwR-projectorg/

ReEves, P. G. \& DeMARS, L. C. (2004). Copper deficiency reduces iron absorption and biological half-life in male rats. Journal of Nutrition 134, 1953-1957.

Reeves, P. G., DeMars, L. C., Johnson, W.T. \& LuKAski, H. C. (2005). Dietary copper deficiency reduces iron absorption and duodenal enterocyte hephaestin protein in male and female rats. Journal of Nutrition 135, 92-98.

Relling, D. P., Esberg, L. B., Johnson, W. T., Murphy, E. J., Carlson, E. C., Lukaski, H. C., SAARI, J. T. \& REN, J. (2007). Dietary interaction of high fat and marginal copper deficiency on cardiac contractile function. Obesity 15, 1242-1257.

Russell, W. M. S. \& BurCH, R. L. (1959). The Principles of Humane Experimental Technique. Methuen, London.

RYAN, L. (1993). Using historical control data in trend tests for clustered binary data. Biometrics 49, 1126-1135.

SAari, J. T., ReEves, P. G., Johnson, W. T. \& Johnson, L. K. (2006). Pinto beans are a source of highly bioavailable copper in rats. Journal of Nutrition 136, 2999-3004.

SCHUlz, K. F. \& GRIMES, D. A. (2005). Sample size calculations in randomised trials: mandatory and mystical. Lancet 365, 1348-1353.

SPiegelhalter, D. J., Abrams, K. R. \& Myles, J. P. (2004). Bayesian Approaches to Clinical Trials and Health-care Evaluation. John Wiley \& Sons Ltd., Chichester, UK.

Thomas, N. (2008). Historical control. Wiley Encyclopedia of Clinical Trials 1-3. 
Viele, K., Berry, S., Neuenschwander, B., Amzal, B., Chen, F., Enas, N., Hobbs, B., Ibrahim, J. G., Kinnersley, N., LindBorg, S., MicAlleF, S., Roychoudhury, S. \& ThOMPSON, L. (2014). Use of historical control data for assessing treatment effects in clinical trials.

Pharmaceutical Statistics 13, 41-54.

Wheeler, D. J. \& Chambers, D. S. (1992). Understanding Statistical Process Control, 2nd ed. SPC Press, Knoxville, Tennessee.

YANAGAWA, T. \& HoEL, D. G. (1985). Use of historical controls for animal experiments.

Environmental Health Perspectives 63, 217-224.

Yoshimura, I. \& MATSUmoto, K. (1994). Notes on the use of historical controls. Environmental Health Perspectives 102 Suppl 1, 19-23.

\section{APPENDIX}

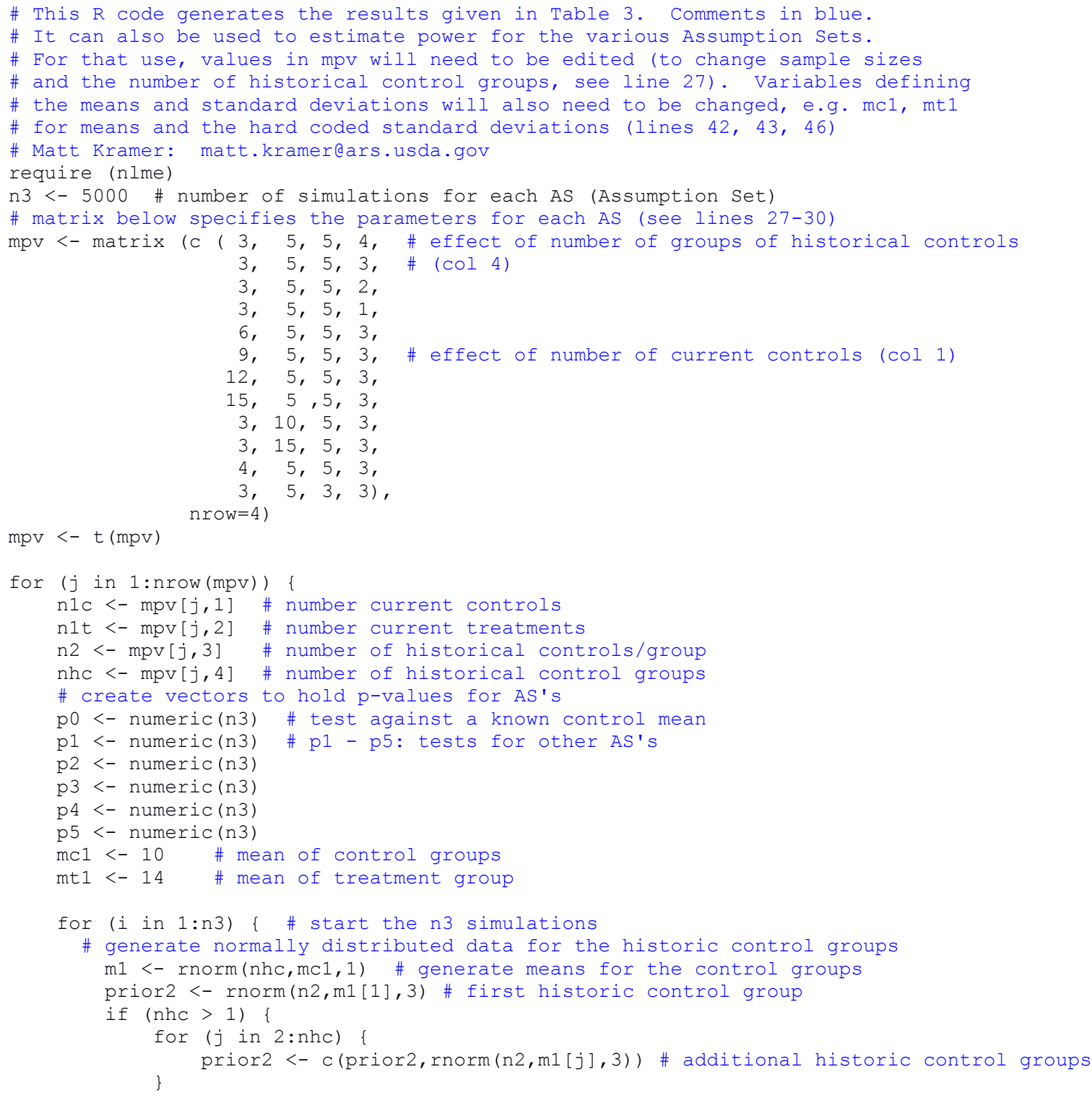


\} expla <- rep (letters [2: $(\mathrm{nhc}+1)]$, each=n2) \# start from b

currentla <- c(rnorm(n1t, mt1,3), rnorm(n1c,mc1,3)) \# generate current treatments, $\exp 2 a<-\operatorname{rep}(" a ", n 1 t+n 1 c)$

\# controls

exp2b <- c (rep ("A", n1t), rep ("C", nhc*n2 + n1c)) \# use A for treatment to make \# it first

all1a <- data.frame $(\exp =\mathrm{c}(\exp 2 \mathrm{a}, \exp 1 \mathrm{a})$, treat=exp2b, y=c (current1a, prior2) \# create data.frame

all1a\$exp.treat <- factor(paste (all1a\$exp, allla\$treat, sep="."))

\# create factor levels

all1b <- groupedData (y exp.treat | exp, data=all1a) \# make a grouped data set \# contrast treatment vs all controls, allow for experiment effect, variances equal fitla $<-$ lme (y $\sim$ treat, random $=\sim 1$ exp, data=allib)

\} \# end: various combinations of current treatment, current control, historical controls 